\title{
cDNA microarray analysis of invasive and tumorigenic phenotypes in a breast cancer model
}

\author{
Harriet M Kluger ${ }^{1}$, Yuval Kluger ${ }^{2}$, Maureen Gilmore-Hebert ${ }^{3,5}$, Kyle DiVito ${ }^{6}$, Joseph T \\ Chang $^{4}$, Sofya Rodov ${ }^{3}$, Olga Mironenko ${ }^{6}$, Barry M Kacinski ${ }^{3}$, Archibald S Perkins ${ }^{6,8}$ and \\ Eva Sapi ${ }^{5,7,8}$
}

${ }^{1}$ Department of Medicine; ${ }^{2}$ Department of Genetics; ${ }^{3}$ Department of Therapeutic Radiology; ${ }^{4}$ Department of Statistics; ${ }^{5}$ Department of Obstetrics and Gynecology; ${ }^{6}$ Department of Pathology, Yale University School of Medicine, New Haven, CT, USA and ${ }^{7}$ Department of Biology and Environmental Sciences, University of New Haven, West Haven, CT, USA

\begin{abstract}
The fms oncogene encodes the macrophage colony-stimulating factor receptor (CSF1R), a transmembrane tyrosine kinase receptor, which is abnormally expressed in breast cancer. Transfection of wild-type CSF1R into HC11 mammary epithelial cells (HC11-CSF1R) renders the transfectants capable of in vitro local invasion and in vivo tumorigenesis. Transfection with CSF1R mutated to express phe at the tyr-721 autophosphorylation site (HC11-CSF1R-721) creates a phenotype that lacks metastastic competence but maintains local invasiveness. Conversely, HC11 cells transfected with CSF1R mutated at tyr-807 (HC11-CSF1R-807) retain their metastatic competence, but are not locally invasive. Our aims were to determine which genes were differentially expressed with transfection of HC11 with wild-type CSF1R, and to determine the effect of mutation at the autophosphorylation sites on gene expression, using $4.6 \mathrm{~K}$ cDNA microarrays. Complementary DNA from HC11, HC11CSF1R-721 and HC11-CSF1R-807 were each hybridized together with HC11-CSF1R on individual arrays. A principal component spectral method combined with prenormalization procedures was used for sample clustering. Differentially expressed genes were identified by the analysis of variance. Confirmation by Northern blotting was performed for MAP kinase phosphatase-1, WDNM1 (extracellular proteinase inhibitor), Trop 2 (tumor-associated calcium signal transducer-2), procollagen type IV alpha, secretory leukoprotease inhibitor, prenylated snare protein Ykt6, ceruloplasmin and chaperonin 10. Many of these genes have not previously been associated with tumor invasion and metastasis. We have successfully identified genes that can be linked to the invasive phenotypes or to tumorigenesis. These genes provide a basis for further studies of metastatic progression and local invasiveness, and can be evaluated as therapeutic targets.

Laboratory Investigation (2004) 84, 320-331, advance online publication, 19 January 2004; doi:10.1038/labinvest.3700044
\end{abstract}

Keywords: cDNA microarrays; CSF1R; invasion; tumorigenicity; gene expression profiles

The cfms proto-oncogene encodes the only known receptor for the macrophage colony-stimulating factor (CSF1). CSF1R is a transmembrane tyrosine kinase receptor, and its ligand, CSF1, has soluble, membrane-bound and cell matrix-associated isoforms. ${ }^{1-3}$ The CSF1R/CSF1 receptor/ligand pair has essential physiologic functions in monocyte and macrophage differentiation, ${ }^{4,5}$ embryonic implanta-

Correspondence: HM Kluger, Section of Medical Oncology WWW211, Yale University School of Medicine, 333 Cedar Street, New Haven, CT 06520, USA.

E-mail: Harriet.Kluger@Yale.edu

${ }^{8}$ These authors contributed equally to this work.

Received 01 August 2003; revised 18 November 2003; accepted 21

November 2003; published online 19 January 2004 tion and placental development, and lactogenic differentiation of the human breast. ${ }^{6-8}$ Abnormally high CSF1R expression has clinically been associated with aggressive breast, ovarian, endometrial, and prostate cancer. ${ }^{9-16}$

Most invasive breast carcinoma cells express readily detectable levels of activated CSF1R. ${ }^{13}$ Studies $^{17}$ have shown that invasive breast cancer cells coexpress the ligand CSF 1, but adjacent in situ carcinoma cells do not. Moreover, in early-stage breast cancer patients treated with local therapy only, high levels of CSF1R expression also have been associated with a higher likelihood of ipsilateral recurrence. ${ }^{18}$

To further evaluate the role of CSF1R in invasion and metastasis, we studied a mouse cell line model ${ }^{19}$ that utilized the HC11 cell line, a spontaneously 
immortalized mammary epithelial cell line cultured from lactating BALB/c mice, which expressed high levels of CSF1. When HC11 was stably transfected with the mouse wild-type CSF1R oncogene, coexpression of the endogenous ligand produced an autocrine system in all transfectants. Compared with the parental HC11 cells, transfectants expressing the wild-type CSF1R (HC11-CSF1R) invaded 100 -fold more efficiently through a barrier reconstituted basement membrane (Matrigel). Moreover, the transfectants were capable of forming colonies in soft agar. When HC11-CSF1R cells were injected intravenously in mice, they produced 10 times more lung metastases than the parental HC11 cell line.

At least six sites of tyrosine autophosphorylation have been observed after the activation of CSF1R, of which two (tyr-721 and tyr-807) have been shown to be important for coupling the CSF1R to intracellular signal transduction pathways. ${ }^{20,21}$ The tyr-807 phosphorylation site plays a critical role in CSF1dependent differentiation in monocytes, and tyr807 phosphorylation is required for binding of elements of the STAT1 and Src pathways, ${ }^{22}$ and the activation of the ras-GAP pathway. ${ }^{23}$ Phosphorylation of tyr-721 is required for the binding of the p100 subunit of PI3-kinase and for CSF1-dependent mitogenesis. ${ }^{24}$ CSF1R was mutated tyr- $>$ phe individually at these two autophosphorylation sites. ${ }^{20}$ When the HC11 cells were transfected with CSF1R mutated at the 721 phosphorylation site, we obtained a cell line (HC11-CSF1R-721) with high capability for in vitro protease production and local invasion, but no capacity for anchorage-independent growth and in vivo metastasis after the intravenous injection. ${ }^{19}$ Conversely, transfection of HC11 cells with CSF1R mutated at the 807 phosphorylation site created a phenotype (HC11-CSF1R807) incapable of in vitro invasion, but capable of anchorage-independent growth and capable of producing in vivo metastasis when injected intravenously into mice. ${ }^{19}$ Confirmation of presence of high levels of CSF-1R in these cell lines has been performed by Northern and Western blots. ${ }^{19}$

cDNA microarray technology enables us to examine patterns of expression of thousands of genes simultaneously. ${ }^{25-27}$ We hypothesize that transfection of HC11 cells with CSF1R and with the two modified receptors discussed above induces changes in the expression of numerous genes associated with invasion and tumorigenesis. Our goals were: (1) to identify genes that were upregulated or downregulated with transfection of HC11 with wild-type CSF1R and (2) to identify gene expression changes associated with mutation of CSF1R at the 721 and 807 autophosphorylation sites.

We have pursued our goals using this unique cell line model that enables us to compare gene expression profiles in a spontaneously immortalized parental cell line with those observed after transfection with a single gene, the wild-type or mutated CSF1-R. Other cell lines such as BT20 and SKBR3, which are human cell lines, also express CSF1 and CSF1R. However, these cell lines lack counterparts that are isogenic except the CSF-1R expression, and comparisons across different cell lines would reveal gene expression differences that are unrelated to CSF-1R. Therefore, our mouse model serves as a basis for further studies in human cell lines and in human tissues. Furthermore, our model enables us to study changes in gene expression caused by point mutation of critical autophosphorylation sites in CSF1R, necessary for the activation of specific downstream signal transduction pathways.

\section{Materials and methods}

\section{Cell Lines and Cell Culture}

HC11 cells originated from mammary glands of BALB/c mice in mid-pregnancy as described by Hynes et al. ${ }^{28} \mathrm{HC} 11$ had been stably transfected with the pcDNA3 expression vector encoding cDNAs for either the wild-type CSF1R or the Tyr- $>\mathrm{Phe}^{721}$ mutation or the Tyr- $>\mathrm{Phe}^{807}$ mutation as described previously. ${ }^{19}$ We selected the clones that invaded most efficiently through the barrier reconstituted basement membrane (Matrigel) in our previous experiments. All four cell lines (HC11, HC11CSF1R, HC11-CSF1R-721, and HC11-CSF1R-807) were incubated at $37^{\circ} \mathrm{C}$, with $5 \% \mathrm{CO}_{2}$ in DME/F12 medium (Sigma Chemical Co, St Louis, MO, USA) containing 10\% fetal bovine serum (Life Technologies Inc.), $5 \mu \mathrm{g} / \mathrm{ml}$ bovine insulin (Sigma Chemical Co.), and $10 \mathrm{ng} / \mathrm{ml}$ murine epidermal growth factor (Sigma). HC11 cells that had been transfected with either the wild type or the mutant types of CSF1R were maintained in media containing $300 \mu \mathrm{g} / \mathrm{ml}$ gentamicin sulfate (Gemini Bio-Products, Woodland, CA, USA).

\section{RNA Extraction}

Total RNA was extracted from the four cell lines using the guanidine-cesium chloride method. ${ }^{19}$ The samples were electrophoresed in a $1 \%$ agarose gel containing $2.2 \mathrm{M}$ formaldehyde to verify the integrity of the RNA.

\section{Probe Preparation and Labeling}

Probe preparation and labeling were performed as described previously. ${ }^{29}$ Briefly, $25 \mu \mathrm{g}$ of total RNA was used for each cell line for each array. cDNA probes were synthesized by reverse transcription using Superscript RT (Gibco, Tulsa, OK, USA) with oligo-dT as a primer, incorporating allyl aminedeoxyuridine triphosphate (aa-dUTP; Sigma) into synthesized cDNA. Coupling of cyanine-3 (cy3) or cyanine-5 (cy5) dyes to aa-dU-modified cDNAs was 
carried out using NHS-ester cy-3 or cy-5 dye (Pharmacia, New York, NY, USA) by incubation at $25^{\circ} \mathrm{C}$ for $90 \mathrm{~min}$ in subdued light.

\section{cDNA Microarray Slide Hybridization}

Microarray slides of (4.6 K cDNA) were purchased from the Keck Microarray Facility at Yale University. A full list of genes included in this microarray is available upon request. Hybridization of fluorescently labeled probes to the glass slides was performed with hybridization buffer (50\% deionized formamide, $12.5 \%$ SSPE, $0.625 \%$ SDS, $1.5 \times$ Denhardt's with blockers $(0.5 \mu \mathrm{g} / \mu \mathrm{l}$ mouse Cot 1 DNA, $0.1 \mu \mathrm{g} / \mu \mathrm{l}$ poly-A (15A), $0.2 \mu \mathrm{g} / \mu \mathrm{l}$ yeast tRNA) at $42^{\circ} \mathrm{C}$ for $18-24 \mathrm{~h}$. After hybridization, the slides were washed first with $1 \times \mathrm{SSC} / 0.1 \% \mathrm{SDS}$ at $25^{\circ} \mathrm{C}$ for $15 \mathrm{~min}$, then with $0.2 \times \mathrm{SSC}, 0.1 \% \mathrm{SDS}$, and finally with $0.2 \times$ SSC.

\section{Microarray Slide Scanning}

The slides were scanned with a GSI Lumonics or Axon Model laser scanner. The analysis of the fluorescent hybridization signal of the microarray slide was performed with Genepix software (Axon Instruments, Inc., Union City, CA, USA).

\section{Microarray Experimental Design}

HC11-CSF1R was chosen as the reference sample, to which the other three cell lines were compared. HC11, HC11-CSF1R-721, and HC11-CSF1R-807 were each hybridized four times on arrays with HC11-CSF1R. On half of the arrays HC11-CSF1R was coupled with cy3 and the index sample with cy5, on the other half the dyes were reversed. Each array contains duplicate copies of cDNAs representing the same gene. Two different RNA isolations were used to perform four hybridizations per sample pair, generating eight ratios per gene per sample pair.

\section{Microarray Data Preprocessing}

As a preliminary step in searching for differentially expressed genes between $\mathrm{HC} 11$ (A) and HC11CSF1R (B), we excluded genes for which we did not have good hybridization in at least one of the eight measurements. Similarly, in comparing HC11CSF1R-721 (C) or HC11-CSF1R-807 (D) cell lines to HC11-CSF1R (B), we analyzed genes that have eight complete measurements.

Genes that had complete sets of 24 ratios with expression levels greater than 100 pixels per spot from these sets of experiments were selected for a principal component data visualization technique and for unsupervised clustering. In each of the 24 experiments, gene expression levels of the index sample (A, C and D) and of the reference sample (B) were normalized by the median expression level of all genes in that sample. These normalized expression levels were then used to calculate gene expression ratios between the index and reference sample. For projection of the data onto a pair of the best partitioning gene principal components (described below), we selected a subset of genes that satisfied the above filtering procedure (over 2500 genes), and had at least two-fold expression changes in one or more of the 24 ratios.

\section{Differentially Expressed Genes}

To identify the differentially expressed genes, we applied the analysis of variance (ANOVA) ${ }^{30}$ package provided by the Jackson Laboratory (http:// www.jax.org/research/churchill/software/anova/ index.html).

We used the dye-swap design to assess the differential expression between HC11 and HC11CSF1R. Similarly, we compared the differential expression of HC11-CSF1R-721 and HC11-CSF1R807 with HC11-CSF1R. In the terminology of Kerr et $a l,{ }^{30}$ the four cell lines in our experiments are referred to as 'varieties'. The ANOVA model partitions the variation in gene expression over genes and varieties into a number of effects, attempting to isolate the effects of interest while appropriately adjusting for other systematic effects. For example, the model includes an average effect for each gene and an average effect for each variety. The model also includes gene-variety interactions, which are the parameters of primary interest. These genevariety interactions model the different effects of the varieties for each individual gene. Other effects, such as systematic differences between the dyes, were accounted for in other parameters in the ANOVA. We used 20000 iterations of the bootstrap to calculate confidence intervals for the genevariety interactions. This use of the bootstrap provides results that are more robust than $t$-tests and $t$ confidence intervals, when error distributions are skewed or long-tailed or otherwise non-normal. ${ }^{30}$ Our criterion for selecting genes that were upregulated (or downregulated) relative to HC11CSF1R was that both end points of their $99 \%$ confidence intervals be positive (or negative, respectively). We thus generated three lists of genes that are differentially expressed with respect to HC11-CSF1R. The eight ratios per sample pair for each gene were then combined to form a single estimate of the true ratio.

\section{Microarray Data Analysis}

In order to verify that the 24 sets of ratios are separable into three distinctive groups despite the sample similarity, we visualized their distribution in a reduced two-dimensional space. For dimension 
reduction, we used the method of principal components to project the data onto a lower dimensional space spanned by the principal component direction vectors, which are the linear combinations of the expression ratios of the various genes that capture most of the variance of the complete data.

This projection is based on singular value decomposition, coupled with a preprocessing normalization step, as described in recent work on simultaneous clustering of genes and samples. ${ }^{31}$ We selected a particular normalization procedure involving a repeated row and column normalization of the matrix representing the data (whose rows correspond to genes and columns correspond to the 24 ratios mentioned above). This two-way rescaling was designed to factor out overall gene and sample effects. In this case, we projected the data onto the second and third principal components because the normalization procedure causes the first principal component to be uninformative. An alternative normalization procedure that leads to similar results and is suitable for extracting a gene-sample interaction matrix is obtained by taking the log transformation of the data matrix followed by the subtraction of the row and column means and the addition of the overall mean.

\section{Northern Blot Analysis for Confirmation of Gene Expression}

Northern blot analysis was performed as described previously. ${ }^{19}$ Briefly, total RNA $(10 \mu \mathrm{g})$ was electrophoresed on a $1 \%$ agarose/ $/ 4 \mathrm{M}$ formaldehyde gel, and was blotted onto a nylon membrane. Ethidium bromide staining of RNA was used as a loading control. Filters were prehybridized for $1 \mathrm{~h}$ in a PerfectHyb Plus hybridization buffer (Sigma-Aldrich Co, St Louis, MO, USA) at $68^{\circ} \mathrm{C}$ with salmon sperm DNA (Sigma). Hybridization was performed with the same hybridization buffer and ${ }^{32} \mathrm{P}$-labeled DNA probes (ResGen, Carlsbad, CA, USA) at $68^{\circ} \mathrm{C}$ for $16 \mathrm{~h}$. Signals on the washed filter were visualized by Kodak XAR-5 film autoradiography.

\section{Cell Line Tissue Microarray Construction and Immunohistostaining}

SKBR-3, BT-20, BT-549, BT-474, MDA-MB-468, and MDA-MB-435S cell lines were purchased from the American Type Culture Collection (Manassas, VA, USA), and grown per ATCC recommendations (http://www.atcc.org/). Tissue microarrays containing the cell blocks were made as described elsewhere ${ }^{32}$ In all, 10 cores measuring $0.6 \mathrm{~mm}$ per cell line were obtained and spaced $0.8 \mathrm{~mm}$ apart on the tissue microarrays. Immunohistostaining of the tissue arrays was performed for the products of a subset of genes for which there were commercially available antibodies. For mouse and rabbit primary antibodies, we used a standard protocol established in the Department of Pathology. ${ }^{33}$ The protocol was modified for goat primary antibodies by using a biotinylated secondary anti-goat IgG (Vector Laboratories, Inc., Burlingame, CA, USA) followed by washing with phosphate-buffered saline and incubation for $30 \mathrm{~min}$ with ABC complex (Vector Laboratories, Inc.). The rest of the staining procedure was as described. ${ }^{33}$ Slides were stained for CSF-1R (mouse anti-human IgG at 1:2000, Santa Cruz Biotechnology, Santa Cruz, CA, USA), secretory leukoprotease inhibitor (goat anti-human IgG at 1:500, R\&D Systems, Inc., Minneapolis, MN, USA), MAP kinase phosphatase-1 (rabbit anti-human IgG at 1:100, Santa Cruz Biotechnology, Santa Cruz, CA, USA), tumor-associated calcium signal transducer-2 Trop 2 (mouse anti-human IgG at 1:20, BD Biosciences, San Diego, CA, USA), ceruloplasmin (goat anti-human IgG at 1:10 000, Bethyl Laboratories, Inc., Montgomery, TX, USA), chaperonin 10 (rabbit anti-human IgG at 1:10 000, Calbiochem, San Diego, CA, USA), and tissue inhibitor of metalloproteinase 2 (rabbit anti-human IgG, 1:100, Santa Cruz Biotechnologies, Santa Cruz, CA, USA). A cell line tissue microarray was also stained for Her2/neu (rabbit anti-human IgG at 1:300, DAKO Corporation, Carpinteria, CA, USA) to serve as positive and negative controls, utilizing the published data on Her2/neu expression in these cell lines.

\section{Results}

We studied gene expression profiles in our four cell lines: the parental cell line HC11 (A), HC11 transfected with CSF1R (B), HC11 transfected with CSF1R at the 721 phosphorylation site (C), and HC11 transfected with CSF1R mutated at the 807 phosphorylation site (D), using a $4.6 \mathrm{~K}$ mouse cDNA microarray. The reference sample, to which the other three cell lines were compared, was HC11CSF1R. The index samples, HC11, HC11-CSF1R721, and HC11-CSF1R-807, were each hybridized four times on arrays with HC11-CSF1R. Each array contains duplicate spots of cDNAs representing the same gene, thus the four arrays per sample pair produced eight data points per ratio per gene, resulting in a total of 24 ratios per gene from the three reference samples.

After preprocessing the data as described above, we projected the gene expression profiles of the eight data sets for the three index samples relative to the reference sample onto a pair of leading gene principal components. As demonstrated in the scatter plot shown in Figure 1, the different samples are clearly separable. Separation of these samples was also seen using our spectral bidirectional clustering method. ${ }^{31}$

Table 1 includes genes that consistently revealed differential expression of 1.8 -fold or more between one or more of the index cell lines and HC11-CSF1R, with a confidence interval of $99 \%$. The values 


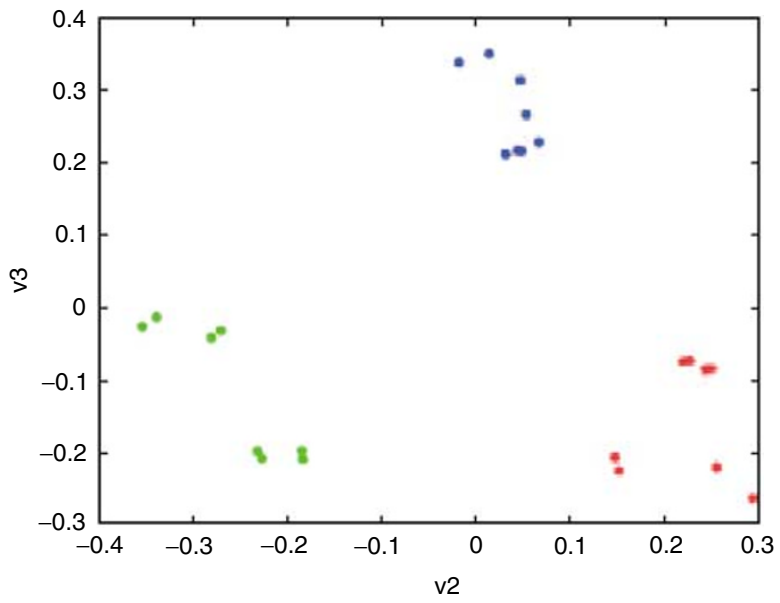

Figure 1 Projection of the samples onto a subspace spanned by two principal components. Clustering of samples is evident, even though the identities of the samples were not used in performing the projection. The data matrix was preprocessed by an iterative procedure of rescaling rows and columns, leading to a normalized matrix having all row sums equal and all column sums equal. Using the normalized matrix, projections of the samples onto the second and third principal components for expression ratios of HC11(A) to HC11-CSF1R (B) are shown by red dots, ratios of HC11-CSF1R-721(C) to HC11-CSF1R by blue dots, and ratios of HC11-CSF1R-807(D) to HC11-CSF1R by green dots.

shown are based on the gene-variety interactions estimated by the ANOVA. The numbers represent the log ratio (base 2) of the index sample to HC11CSF1R. The genes have been sorted by Gene Ontology Molecular Function groups (http:// www.geneontology.org).

Table 2 includes some of the genes that consistently revealed differential expression between the parental cell line HC11 and HC11-CSF1R, with a confidence interval of 99\%. Likewise, Table 3 lists genes that were differentially expressed when CSF1R was mutated at the 721 phosphorylation site compared with HC11-CSF1R. Differential expression of these genes correlates with the loss of ability for anchorage-independent growth and formation of metastases when injected intravenously into mice. Table 4 includes genes that consistently revealed differential expression with a confidence interval or 99\% when comparing HC11-CSF1R-807 with HC11CSF1R. Differential expression of these genes correlates with the loss of ability for in vitro invasion.

Genes that had at least a two-fold difference in the expression based on the microarray analysis, and for which cDNA probes were readily available, were chosen for confirmatory Northern blot analysis. These included chaperonin 10, procollagen type IV alpha, MAP kinase phosphatase, prenylated snare protein, secretory leukoprotease inhibitor, Trop 2, WDNM1, and ceruloplasmin as shown in Figure 2. RNA levels in the Northern blots were consistent with the microarray data.

Immunohistostaining was performed for a subset of genes using a tissue microarray containing nonisogenic human breast cancer cell lines. The staining patterns are summarized in Table 5 . Examples of negative, weak and strong staining are shown in Figure 3.

\section{Discussion}

In this communication, we present data on changes in gene expression observed after mouse HC11 mammary epithelial cells were transfected with either a wild-type CSF1R gene or one of two tyr to phe point mutated versions, which interrupted specific downstream signal transduction pathways. Microarray analysis was employed to quantitate the differences in gene expression among the four cell lines. The gene expression changes presented above were consistent across numerous repetitions, using cells harvested at different times. Moreover, the results were consistent with the Northern blot analysis performed on a subset of genes. As detailed below, some of the changes in gene expression were as expected, based on information about these genes in the literature (eg MAP kinase phosphatase, tissue inhibitor of metalloproteinase 2), and other genes are 'novel' in terms of their involvement in tumor invasion and metastasis.

As a first step in the analysis of the changes in gene expression profiles in our cell lines, we performed a clustering analysis. We were successful in clustering the samples in an unsupervised fashion, which succeeded despite sample similarity (all cell lines having originated from the same parental cell line and having been transfected with a single gene or one of two point mutated variants).

The microarrays used in this study contained probes for 4600 genes representing a broad spectrum of cellular functions, as well as numerous expressed sequence tags. Other investigators have used varying sizes of microarrays to analyze gene expression profiles in breast cancer specimens, some with hundreds of genes and some with thousands. ${ }^{34-38}$ Comparisons between our data set and other published data sets are rather difficult to make, because the different experimental models studied have been analyzed on arrays that include different gene sets and different species. Moreover, most publications discuss only the subset of genes that were differentially expressed, and do not focus on genes that might have been included on the array, but were not differentially expressed. Some of the differentially expressed genes in our gene set have not previously been associated with aggressive malignant behavior. Many of the other breast cancer cell line microarray analyses published in the literature make comparisons between completely different cell lines, ${ }^{35,39-42}$ which is problematic both methodologically and fundamentally, since many of the differences between cell lines may represent differences that are unrelated to invasiveness or tumorigenesis. We compare a spontaneously 
Table 1 Differentially expressed genes

Chaperone proteins

Heat-shock $10 \mathrm{kDa}$ protein 1 (chaperonin 10)

Immunity proteins

Complement component 3

Immunoresponsive gene 1

Catalytic proteins

Cyclin-dependent kinase inhibitor 1A

Ceruloplasmin

Carboxypeptidase X2

EST highly similar to AMP deaminase 2

MAP kinase phosphatase-1

Protein kinase, cGMP dependent, type II

Cytochrome P450IIIA25

EST highly similar to serine protease

Mitogen-activated protein kinase kinase kinase kinase 1

Signal tranducer proteins

EST

Src-like adaptor 2

Tumor-associated calcium signal transducer 2 (Trop 2)

Structural molecules

Procollagen type IV alpha 1

Tubulin, alpha 4

Claudin 3

Ribosomal protein L19

Transporter proteins

ATPase-like vacuolar proton channel

Binding proteins

Naked cuticle 1 homolog (Drosophila)

Groucho-related gene 1 protein (Grg1)

Tripartite motif protein 13

Enzyme regulatory proteins

Secretory leukoprotease inhibitor

Tissue inhibitor of metalloproteinase 2

WDNM1/extracellular proteinase inhibitor

Unknown function/other

Calpain small subunit

EST, mod. similar to hypothetical protein DJ667H12.2

Interferon-activated gene 203

EST

Tenascin C

EST weakly similar to WDNM1 protein precursor

EST similar to hypothetical protein similar to beta-transducin family

Oxoglutarate dehydrogenase (lipoamide)

EST

Semaphorin Y

EST

Stromal cell-derived factor receptor 1

EST

EST

EST

EST

Paired related homeobox 1

EST

Treacher Collins Franceschetti syndrome

EST

EST

EST

EST

EST
Serine (or cysteine) proteinase inhibitor, clade H, member 1

Prenylated SNARE protein Ykt6 (Ykt6)

\begin{tabular}{|c|c|c|c|}
\hline AI326777 & 0.0 & 0.2 & 1.2 \\
\hline AI327085 & -1.2 & -0.9 & -0.3 \\
\hline AI528519 & -0.7 & -1.2 & -1.6 \\
\hline AI323667 & -1.0 & -1.1 & -1.3 \\
\hline AI323806 & 0.0 & 0.4 & 0.8 \\
\hline AI323769 & -0.5 & -1.0 & -1.7 \\
\hline AI326771 & 0.3 & 0.2 & 1.0 \\
\hline AI450899 & -0.6 & -1.2 & -0.4 \\
\hline AI325917 & 1.1 & 0.4 & 1.0 \\
\hline AI 447755 & -0.6 & -1.0 & -0.4 \\
\hline AI327008 & 0.0 & -0.9 & -1.0 \\
\hline AI452270 & 0.3 & 0.0 & 0.8 \\
\hline AI413994 & 0.0 & -0.5 & -0.8 \\
\hline AI447805 & -1.3 & -1.2 & -1.7 \\
\hline AI430952 & 0.5 & 0.9 & 0.6 \\
\hline AI324175 & -1.3 & -0.6 & -0.7 \\
\hline AI528741 & 1.0 & 0.0 & 0.1 \\
\hline AI325223 & -0.6 & -1.0 & 0.1 \\
\hline AI414705 & -0.8 & -0.9 & -1.4 \\
\hline AI324702 & -0.7 & -1.4 & -0.7 \\
\hline AI385732 & 0.5 & 1.0 & 0.5 \\
\hline AI426390 & 0.5 & 1.1 & 0.7 \\
\hline AI324893 & -0.8 & -1.8 & -1.7 \\
\hline AI449123 & 0.9 & -0.1 & 0.1 \\
\hline AI324948 & -0.8 & -1.8 & -2.1 \\
\hline AI426775 & 1.0 & 0.3 & 0.3 \\
\hline AI385534 & 1.0 & 1.1 & 0.0 \\
\hline AI324827 & -0.8 & -1.8 & -2.0 \\
\hline AI430908 & 0.4 & 1.1 & 0.6 \\
\hline AI413620 & 0.2 & 0.7 & 1.0 \\
\hline AI451137 & 0.2 & -1.1 & -0.5 \\
\hline AI447310 & 0.1 & 0.9 & 0.7 \\
\hline AI327207 & 0.0 & 0.6 & 1.0 \\
\hline AI451408 & 0.0 & -1.4 & 0.0 \\
\hline AI893430 & -0.7 & -1.3 & -0.7 \\
\hline AI324749 & -0.9 & -0.3 & -0.4 \\
\hline AI450057 & -1.0 & -1.2 & 0.0 \\
\hline AI451533 & -1.0 & 0.0 & -0.2 \\
\hline AI450885 & -1.1 & -0.9 & -0.2 \\
\hline AI324156 & -1.3 & -0.4 & -0.7 \\
\hline AI451155 & -1.6 & -1.5 & -1.5 \\
\hline AI447770 & 1.1 & 0.0 & 0.0 \\
\hline AI429580 & 0.4 & 1.1 & 0.0 \\
\hline AI452305 & 0.3 & -0.9 & 0.8 \\
\hline AI426130 & 0.0 & 0.0 & 0.9 \\
\hline AI450779 & -0.9 & 0.0 & 0.2 \\
\hline AI451115 & -0.9 & -0.9 & -0.2 \\
\hline AI449474 & -1.0 & -0.5 & -0.3 \\
\hline AI449074 & -1.1 & -0.0 & 0.1 \\
\hline AI426662 & -1.2 & -0.3 & -1.1 \\
\hline AI449936 & -1.3 & -0.5 & -1.2 \\
\hline AI415183 & -0.6 & -1.1 & -1.1 \\
\hline
\end{tabular}

Log ratios of 52 genes that were consistently differentially expressed (99\% confidence interval) in either HC11 (A), HC11-CSF1R-721 (C) or HC11CSF1R-807 (D) relative to the reference sample, HC11-CSF1R (B). The genes have been sorted by functional Gene Ontology groups. A positive value reflects a higher level of expression in the index sample than in HC11-CSF1R, and vice versa for a negative value. 
Table 2 Genes that are differentially expressed with stable transfection of HC11 with CSF1R

\begin{tabular}{ll}
\hline Higher in HC11 than HC11-CSF1R & Lower in HC11 than HC11-CSF1R \\
\hline MAP kinase phosphatase-1 & Prenylated SNARE protein Ykt6 (Ykt6) \\
EST, AI447770 & EST, AI450779 \\
Procollagen type IV alpha 1 & Treacher Collins Franceschetti syndrome \\
WDNM1/extracellular proteinase inhibitor & Oxoglutarate dehydrogenase (lipoamide) \\
Tissue inhibitor of metalloproteinase 2 & Immunoresponsive gene 1 \\
Tripartite motif protein 13 & EST, AI450057 \\
& Semaphorin Y \\
& EST AI449474 \\
& EST, AI449074 \\
& EST, AI450885 \\
& Heat-shock 10 kDa protein 1 (chaperonin 10) \\
& EST, AI426662 \\
EST, AI449936 \\
Tumor-associated calcium signal transducer 2 (trop 2 gene) \\
EST, AI447805 \\
Stromal cell-derived factor receptor 1 \\
EST, AI451155 \\
Secretory leukoprotease inhibitor
\end{tabular}

A list of 24 genes that were consistently differentially expressed (99\% confidence interval) in HC11-CSF1R relative to HC11.

Table 3 Genes that are differentially expressed with mutation of CSF1R at the 721 phosphorylation site

\begin{tabular}{ll}
\hline Higher in HC11-CSF1R-721 than HC11-CSF1R & Lower in HC11-CSF1R-721 than HC11-CSF1R \\
\hline Naked cuticle 1 homolog (Drosophila) & Claudin 3 \\
EST, AI429580 & Heat-shock 10 kDa protein 1 (chaperonin 10) \\
WDNM1/extracellular proteinase inhibitor & EST, AI452305 \\
Calpain small subunit & Cytochrome P450IIIA25 \\
ATPase-like vacuolar proton channel & EST, AI450885 \\
Src-like-adaptor 2 & Treacher Collins Franceschetti syndrome \\
EST, AI447310 & Protein kinase, cGMP-dependent, type II \\
& Tubulin, alpha 4 \\
& Interferon-activated gene 203 \\
& EST, AI415183 \\
& Immunoresponsive gene 1 \\
& EST, AI450057 \\
& Complement component 3 \\
& EST highly similar to AMP Deaminase 2 \\
& EST, AI447805 \\
Similar to hypothetical protein similar to beta-transducin family \\
EST weakly similar to WDNM1 protein precursor \\
Ribosomal protein L19 \\
EST, AI451155 \\
Groucho-related gene 1 protein (Grg1) \\
Prenylated SNARE protein Ykt6 (Ykt6) \\
Secretory leukoprotease inhibitor
\end{tabular}

A list of 29 genes that were consistently differentially expressed (99\% confidence interval) in HC11-CSF1R-721 relative to HC11-CSF1R.

immortalized cell line with the same cell line after stable transfection with a single oncogene (creating an autocrine system since the parent cell produces the ligand), which alters its invasive and tumorigenic properties. Although HC11 is a mouse cell line, it has been used in numerous model systems to study invasion, tumorigenicity, and anticancer drug testing related to genes known to be involved in human tumor progression such as p53 mutation and ErbB2 transfection. ${ }^{43,44}$

We sorted the genes by Gene Ontology functional classes (Table 1). Many genes encode for catalytic proteins. Other classes include smaller numbers of genes, and many genes and ESTs do not have assigned Gene Ontology classes.

For some genes, our study confirms data found in the literature. For example, WDNM 1 (also known as extracellular proteinase inhibitor) is a gene involved in breast involution. It has been shown ${ }^{45}$ to be downregulated in a metastatic breast phenotype, while being upregulated in a nonmetastatic phenotype. Others have described WDNM1 as a metastasis suppressor gene. ${ }^{46}$ The expression of WNDM1 has recently been shown to be related to p53 dysregulation in breast cell lines. ${ }^{47}$ Our results reveal the downregulation of WDNM1 in HC11 transfected 
Table 4 Genes that are differentially expressed with mutation of CSF1R at the 807 phosphorylation site

Higher in HC11-CSF1R-807 than HC11-CSF1R

MAP kinase phosphatase-1

Serine proteinase inhibitor, clade $\mathrm{H}$, member 1

Carboxypeptidase X2

Tenascin C

EST, similar to hypothetical protein DJ667H12.2

Paired related homeobox 1

EST highly similar to serine protease

Cyclin-dependent kinase inhibitor $1 \mathrm{~A}$
Lower in HC11-CSF1R-807 than HC11-CSF1R

MAP kinase kinase kinase kinase 1

Cytochrome P450IIIA25

EST, AI426662

EST, AI415183

EST, AI449936

Immunoresponsive gene 1

Claudin 3

EST, AI451155

Complement component 3

Ceruloplasmin

EST, AI447805

Groucho-related gene 1 protein (Grg1)

Prenylated SNARE protein Ykt6 (Ykt6)

Secretory leukoprotease inhibitor

A list of 22 genes that were consistently differentially expressed (99\% confidence interval) in HC11-CSF1R-807 relative to HC11-CSF1R.

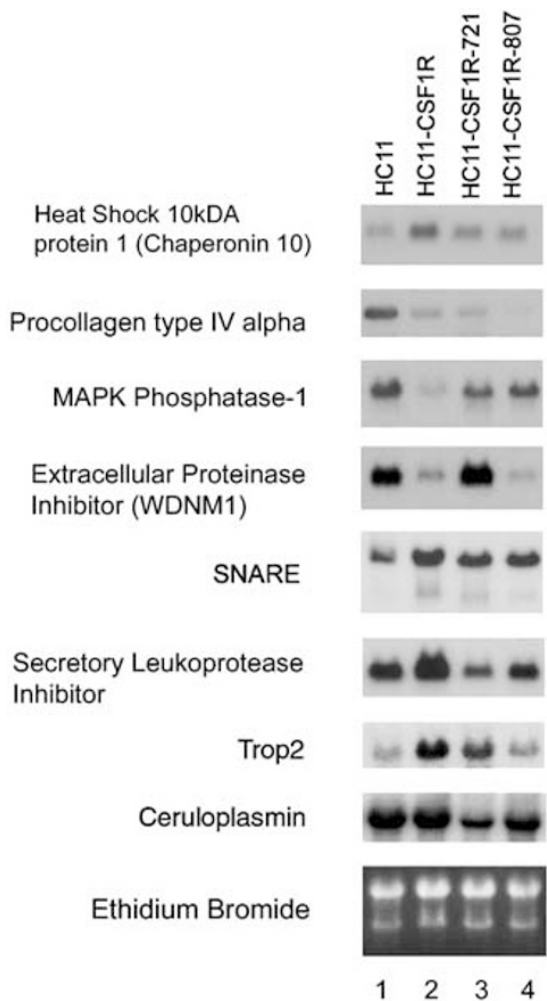

Figure 2 Northern blot analysis of gene expression of selected genes. Total RNA $(10 \mu \mathrm{g})$ was fractionated on $1 \%$ agaroseformaldehyde gels, blotted onto nylon membrane, and hybridized with the probes indicated. The bottom panel represents the $18 \mathrm{~S}$ and 28S RNA in the ethidium bromide-stained agarose gel.

with CSF1R, as well as in HC11 transfected with CSF1R mutated at phosphorylation site 807. However, it was not downregulated in the cell line transfected with the 721 mutation. This suggests that phosphorylation of the 807 site is necessary for the downregulation of WDNM1, and that the downregulation of WDNM1 might be related to tumorigenicity.
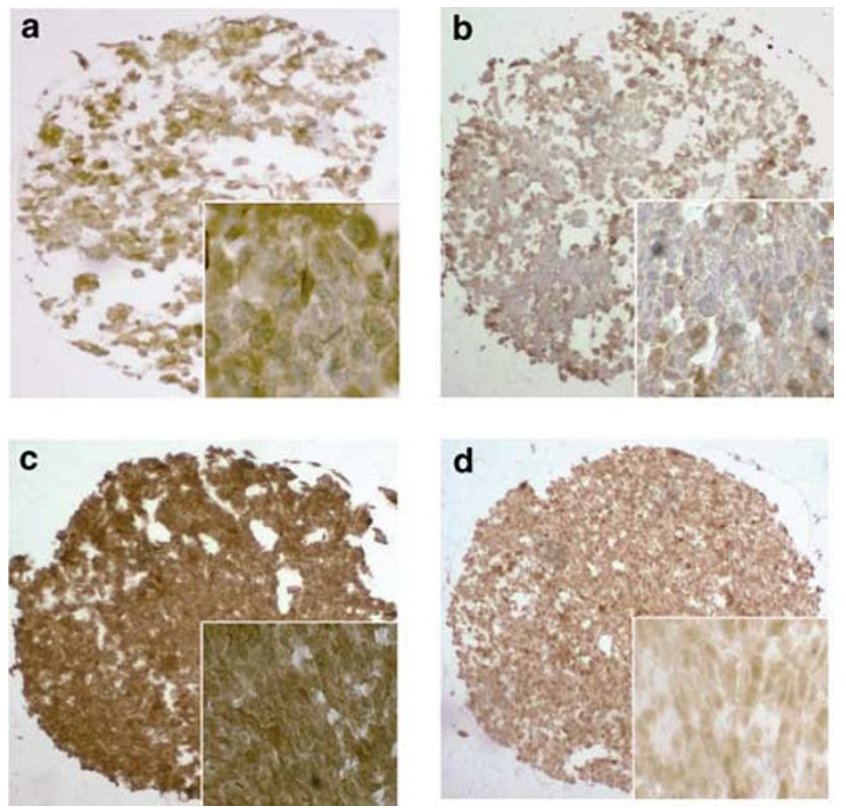

Figure 3 Examples of immunohistostaining of the human cell line tissue micorarray. Immunhistostaining of SKBR-3 was weakly positive for CSF1R (a) and negative for MAP kinase phosphatase1 (b). Immunohistostaining of BT-20 was strongly positive for CSF1R (c) and weakly positive for MAP kinase phosphatase-1 (d).

The MAP kinase phosphatase- 1 expression level was significantly lower in HC11-CSF1R than in HC11. It was not expressed in SKBR-3 cells, and weakly expressed in BT-20 cells. CSF1R activates the RAS, RAF, and MEK pathways, activating MAP kinase. ${ }^{22}$ MAP kinase phosphatase-1 is a downregulator of MAP kinase, and its levels are suppressed when MAP kinase is activated. HC11CSF1R-807 also had a higher expression level of this gene than HC11-CSF1R, suggesting that phosphorylation of tyr-807 is necessary for the activation of MAP kinase. The downregulation of MAP kinase phosphatase-1 in melanocytes by a receptor tyrosine 
Table 5 Immunhistochemical staining of a subset of differentially expressed genes using a human cell line tissue microarray

\begin{tabular}{|c|c|c|c|c|c|c|c|c|}
\hline Cell line & CSF1R & Chaperonin 10 & TIMP-2 & Trop 2 & Ceruloplasmin & $M K P-1$ & SLPI & Her2/neu \\
\hline BT-549 & - & + & - & - & + & - & + & - \\
\hline MDA-MB-435S & - & + & - & + & + & + & + & - \\
\hline MDA-MB-468 & + & ++ & - & - & - & - & - & - \\
\hline SKBR-3 & + & + & + & + & + & - & + & ++ \\
\hline BT-474 & + & + & ++ & ++ & + & + & ++ & ++ \\
\hline BT-20 & ++ & + & + & ++ & ++ & + & ++ & + \\
\hline
\end{tabular}

The immunohistochemical staining of CSF1R, chaperonin 10, TIMP 2, Trop 2, ceruloplasmin, MAP kinase phosphatase-1 (MKP-1), secretory leukoprotease inhibitor (SLPI) and Her2/neu in a number of human breast cancer cell lines. Strongly positive staining is denoted by '++', weakly positive by ' + ' and negative staining is denoted by '- ',

kinase results in suppressed differentiation and a transformed phenotype. ${ }^{48}$ Our results suggest that MAP kinase phosphatase-1 downregulation might be involved in CSF-1R transformation of the HC11 cell line, with the results suggesting that tyr-807 phosphorylation is necessary for this process.

Ceruloplasmin was downregulated in the two cell lines transfected with the mutated CSF1R relative to the wild-type HC11-CSF1R. It is a copper transport protein and an acute phase reactant that has been shown to be upregulated in human breast cancer cell lines ${ }^{49}$ and in human ovarian cancer specimens. ${ }^{50}$ Moreover, ceruloplasmin levels are increased in the serum of patients with breast cancer. ${ }^{51}$ It has been proposed that ceruloplasmin transports iron into malignant cells. ${ }^{50}$ Ceruloplasmin was expressed in most of the human cell lines that we stained. The fact that it is downregulated with mutations of both phosphorylation sites suggests that its downregulation might be mediated through either the PI3 kinase pathway or the MAP kinase pathway or other pathways related to both sites.

Trop 2 (also known as tumor-associated calcium signal transducer) is a calcium signal transducer expressed at high levels by numerous human cancers. $^{52}$ Trop 2 is upregulated in HC11-CSF1R relative to the other cell lines, with a higher level in HC11-CSF1R-721 than in the phenotypes that lack capacity for in vitro invasion (Figure 2). The expression pattern of Trop 2 in most of the human cell lines studied mimicked that of CSF1R (Table 5). The specific role for Trop 2 in breast cancer invasion and metastasis is not known; however, it is a cell surface receptor with a cytoplasmic domain that appears to play a regulatory role in the growth of carcinoma cells. ${ }^{53}$ Our data suggest a role for Trop 2 in tumor invasion.

Tissue inhibitor of metalloproteinase 2 (TIMP 2) was higher in HC11 than in HC11-CSF1R, and was the same in the two cell lines with the mutated CSF1R as in HC11-CSF1R. Previous studies by Sapi et $a l^{19}$ have shown that TIMP 2 inhibits in vitro invasion of HC11-CSF1R. Hence, low levels of TIMP 2 are necessary for HC11-CSF1R to maintain its invasive capabilities. TIMP 2 has also been shown to inhibit the development of mammary tumors in mice,$^{54}$ consistent with our findings.
Secretory leukoprotease inhibitor is a gene with no known role in invasiveness of breast cancer, and warrants further evaluation. The mRNA level of this gene is higher in HC11-CSF1R than in the other cell lines. The protein product of this gene was expressed in most of the invasive human cell lines studied by immunohistostaining. Secretory leukoprotease inhibitor has inhibitory activity against chymotrypsin, trypsin, neutrophil elastase, and cathepsin $G,{ }^{55}$ and has been shown in monocytes to suppress the induction of matrix metalloproteinases. ${ }^{56}$ High expression levels of secretory leukoprotease inhibitor have been found in human ovarian carcinoma specimens. ${ }^{50}$

Procollagen type IV alpha is a precursor to collagen type IV alpha, a component of the basement membrane in mammary glands. Its level was higher in HC11 than in HC11-CSF1R, and the mutation of CSF1R did not change its expression. Previously, we have shown that urokinase expression was upregulated by CSF1R expression in HC11 cell lines. ${ }^{19}$ Urokinase has a major role in the collagenase pathway, therefore our recent findings confirm the involvement of CSF1R in collagen regulation.

Chaperonin 10 (also known as heat shock $10 \mathrm{kDa}$ protein 1) is upregulated in HC11-CSF1R relative to HC11, and is somewhat lower in the cell lines with the mutated CSF1R than in HC11-CSF1R. All of the invasive human breast cell lines studied by immunohistostaining were positive for chaperonin 10. It is involved in mitochondrial protein folding. ${ }^{57}$ Its extracellular homologue, early pregnancy factor, is associated with cell growth and division. ${ }^{58} \mathrm{CSF} 1 \mathrm{R}$ is also upregulated in normal placental trophoblast epithelium. ${ }^{19}$ However, the specific relationship between chaperonin 10 and CSF1R is unknown, and no role for chaperonin 10 in metastasis and invasion was found in the literature.

Based on these data, we have initiated studies to examine the specific roles of some of these genes in invasion and metastasis. We will use siRNA technology to inhibit genes that were upregulated in the transfectants relative to the parental cell line to study whether inhibition of these genes abolishes invasive capability of these cells. Likewise, we will study whether inhibition by siRNA of genes that were upregulated in HC11-CSF1R and HC11-CSF1R-807 
relative to the other cell lines abolishes the ability for anchorage-independent growth and in vivo tumorigenicity. These data serve as the initial step towards understanding the role of CSF-1R, its phosphorylation sites and its downstream genes in breast cancer, and the roles of these genes need to be confirmed in human tumors.

In summary, cDNA microarray analysis of our cell line model reveals a set of genes involved in in vitro invasion and in in vivo tumorigenesis in mice. The reduction of our data into a two-dimensional space shows distinctive clusters of the different samples, despite their similarity. This suggests biological significance of our differential gene expression. Our data confirm some findings in the literature with regard to genes known to be involved in the invasive process. We have also identified novel genes, which have not previously been associated with malignant invasion and metastasis. cDNA microarray analysis of a well-controlled model system involving a highly relevant oncogene is a useful screening tool for identifying novel differentially expressed genes. These genes can provide a basis for further studies of metastatic progression and local invasiveness in human tumors, and can be evaluated as therapeutic targets.

\section{Acknowledgements}

We acknowledge with appreciation the help of Dr Maryann Flick (Department of Therapeutic Radiology, Yale University) for assistance in Northern blot imaging and presentation of the data.

We would like to dedicate this work to our colleague, mentor, and friend, Dr Barry M Kacinski, who passed away unexpectedly on 20 November 2003.

This work was supported by grants from the Ethel F Donaghue Women's Health Investigator Program at Yale, the CJ Swebilius Foundation for Translational Research and the Susan G Komen Breast Cancer Foundation (to HMK), the Anna Fuller Foundation Award for Bioinformatics (to YK) and NIH Grants CA-47292, CA-74832, and the Early Detection Research Network of the National Cancer Institute (to BMK), The Yale Keck cDNA Microarray Facility is supported by the Anna and Argall Hull Fund, the CG Swebilius Trust, NIH Grant NIH 5 U24 DK58776 (to Dr Kenneth Williams, Biotechnical Services, Yale University), and the Department of Pathology, Yale University.

Duality of interests: None declared.

\section{References}

1 Heisterkamp N, Groffen J, Stephenson JR. Isolation of v-fms and its human cellular homolog. Virology 1983;126:248-258.
2 Rettenmier CW, Chen JH, Roussel MF, et al. The product of the c-fms proto-oncogene: a glycoprotein with associated tyrosine kinase activity. Science 1985;228:320-322.

3 Sherr CJ. The fms oncogene. Biochim Biophys Acta 1988;948:225-243.

4 Woolford J, Rothwell V, Rohrschneider L. Characterization of the human c-fms gene product and its expression in cells of the monocyte-macrophage lineage. Mol Cell Biol 1985;5:3458-3466.

5 Sariban E, Mitchell T, Kufe D. Expression of the c-fms proto-oncogene during human monocytic differentiation. Nature 1985;316:64-66.

6 Sapi E, Flick MB, Rodov S, et al. Expression of CSF-I and CSF-I receptor by normal lactating mammary epithelial cells. J Soc Gynecol Invest 1988;5:94-101.

7 Pollard JW, Bartocci A, Arceci R, et al. Apparent role of the macrophage growth factor, CSF-1, in placental development. Nature 1987;330:484-486.

8 Daiter E, Pampfer S, Yeung YG, et al. Expression of colony-stimulating factor-1 in the human uterus and placenta. J Clin Endocrinol Metab 1992;74: 850-858.

9 Kacinski BM, Carter D, Mittal K, et al. High level expression of fms proto-oncogene mRNA is observed in clinically aggressive human endometrial adenocarcinomas. Int J Radiat Oncol Biol Phys 1988;15: 823-829.

10 Kacinski BM, Carter D, Mittal K, et al. Ovarian adenocarcinomas express fms-complementary transcripts and fms antigen, often with coexpression of CSF-1. Am J Pathol 1990;137:135-147.

11 Kacinski BM, Chambers SK, Stanley ER, et al. The cytokine CSF-1 (M-CSF) expressed by endometrial carcinomas in vivo and in vitro, may also be a circulating tumor marker of neoplastic disease activity in endometrial carcinoma patients. Int J Radiat Oncol Biol Phys 1990;19:619-626.

12 Kacinski BM, Scata KA, Carter D, et al. FMS (CSF-1 receptor) and CSF-1 transcripts and protein are expressed by human breast carcinomas in vivo and in vitro. Oncogene 1991;6:941-952.

13 Tang R, Beuvon F, Ojeda M, et al. M-CSF (monocyte colony stimulating factor) and M-CSF receptor expression by breast tumour cells: M-CSF mediated recruitment of tumour infiltrating monocytes? J Cell Biochem 1992;50:350-356.

14 Ramakrishnan S, Xu FJ, Brandt SJ, et al. Constitutive production of macrophage colony-stimulating factor by human ovarian and breast cancer cell lines. J Clin Invest 1989;83:921-926.

15 Ide H, Seligson DB, Memarzadeh S, et al. Expression of colony-stimulating factor 1 receptor during prostate development and prostate cancer progression. Proc Natl Acad Sci USA 2002;99:14404-14409.

16 Baiocchi G, Kavanagh JJ, Talpaz M, et al. Expression of the macrophage colony-stimulating factor and its receptor in gynecologic malignancies. Cancer 1991;67:990-996.

17 Scholl SM, Crocker P, Tang R, et al. Is colonystimulating factor-1 a key mediator of breast cancer invasion and metastasis? Mol Carcinogen 1993;7: 207-211.

18 Maher MG, Sapi E, Turner B, et al. Prognostic significance of colony-stimulating factor receptor expression in ipsilateral breast cancer recurrence. Clin Cancer Res 1998;4:1851-1856. 
19 Sapi E, Flick MB, Rodov S, et al. Independent regulation of invasion and anchorage-independent growth by different autophosphorylation sites of the macrophage colony-stimulating factor 1 receptor. Cancer Res 1996;56:5704-5712.

20 Bourette RP, Myles GM, Carlberg K, et al. Uncoupling of the proliferation and differentiation signals mediated by the murine macrophage colony-stimulating factor receptor expressed in myeloid FDC-P1 cells. Cell Growth Differ 1995;6:631-645.

21 Reedijk M, Liu X, van der Geer P, et al. Tyr721 regulates specific binding of the CSF-1 receptor kinase insert to PI 3'-kinase SH2 domains: a model for SH2mediated receptor-target interactions. EMBO J 1992;11:1365-1372.

22 Bourette RP, Rohrschneider LR. Early events in M-CSF receptor signaling. Growth Factors 2000;17:155-166.

23 Trouliaris S, Smola U, Chang JH, et al. Tyrosine 807 of the v-Fms oncogene product controls cell morphology and association with p120RasGAP. J Virol 1995;69: 6010-6020.

24 van der Geer P, Hunter T. Mutation of Tyr697, a GRB2binding site, and Tyr721, a PI 3-kinase binding site, abrogates signal transduction by the murine CSF-1 receptor expressed in Rat-2 fibroblasts. EMBO J 1993;12:5161-5172.

25 Johnston M. Gene chips: array of hope for understanding gene regulation. Curr Biol 1998;8:R171-R174.

26 Duggan DJ, Bittner M, Chen Y, et al. Expression profiling using cDNA microarrays. Nat Genet 1999;21:10-14.

27 Schena M. Genome analysis with gene expression microarrays. BioEssays 1996;18:427-431.

28 Hynes NE, Taverna D, Harwerth IM, et al. Epidermal growth factor receptor, but not c-erbB-2, activation prevents lactogenic hormone induction of the betacasein gene in mouse mammary epithelial cells. Mol Cell Biol 1990;10:4027-4034.

29 Ma X, Husain T, Peng H, et al. Development of a murine hematopoietic progenitor complementary DNA microarray using a subtracted complementary DNA library. Blood 2002;100:833-844.

30 Kerr MK, Martin M, Churchill GA. Analysis of variance for gene expression microarray data. J Comput Biol 2000;7:819-837.

31 Kluger Y, Basri R, Chang JT, et al. Spectral biclustering of microarray data: coclustering genes and conditions. Genome Res 2003;13:703-716.

32 Hoos A, Cordon-Cardo C. Tissue microarray profiling of cancer specimens and cell lines: opportunities and limitations. Lab Invest 2001;81:1331-1338.

33 Dolled-Filhart M, Camp RL, Kowalski DP, et al. Tissue microarray analysis of signal transducers and activators of transcription 3 (Stat3) and phospho-Stat3 (Tyr705) in node-negative breast cancer shows nuclear localization is associated with a better prognosis. Clin Cancer Res 2003;9:594-600.

34 Sorlie T, Perou CM, Tibshirani R, et al. Gene expression patterns of breast carcinomas distinguish tumor subclasses with clinical implications. Proc Natl Acad Sci USA 2001;98:10869-10874.

35 Zajchowski DA, Bartholdi MF, Gong Y, et al. Identification of gene expression profiles that predict the aggressive behavior of breast cancer cells. Cancer Res 2001;61:5168-5178.

36 Perou CM, Jeffrey SS, van de Rijn M, et al. Distinctive gene expression patterns in human mammary epithe- lial cells and breast cancers. Proc Natl Acad Sci USA 1999;96:9212-9217.

37 Perou CM, Sorlie T, Eisen MB, et al. Molecular portraits of human breast tumours. Nature 2000;406: 747-752.

38 Heiskanen M, Kononen J, Barlund M, et al. CGH, cDNA and tissue microarray analyses implicate FGFR2 amplification in a small subset of breast tumors. Anal Cell Pathol 2001;22:229-234.

39 Schindelmann S, Windisch J, Grundmann R, et al. Expression profiling of mammary carcinoma cell lines: correlation of in vitro invasiveness with expression of CD24. Tumour Biol 2002;23:139-145.

40 Kauraniemi $\mathrm{P}$, Barlund $\mathrm{M}$, Monni O, et al. New amplified and highly expressed genes discovered in the ERBB2 amplicon in breast cancer by cDNA microarrays. Cancer Res 2001;61:8235-8240.

41 Soulez M, Parker MG. Identification of novel oestrogen receptor target genes in human ZR75-1 breast cancer cells by expression profiling. J Mol Endocrinol 2001;27:259-274.

42 Forozan F, Mahlamaki EH, Monni O, et al. Comparative genomic hybridization analysis of 38 breast cancer cell lines: a basis for interpreting complementary DNA microarray data. Cancer Res 2000;60: 4519-4525.

43 Brandt R, Wong AM, Hynes NE. Mammary glands reconstituted with Neu/ErbB2 transformed HC11 cells provide a novel orthotopic tumor model for testing anti-cancer agents. Oncogene 2001;20: 5459-5465.

44 Merlo GR, Basolo F, Fiore L, et al. p53-dependent and p53-independent activation of apoptosis in mammary epithelial cells reveals a survival function of EGF and insulin. J Cell Biol 1995;128:1185-1196.

45 Dear TN, Ramshaw IA, Kefford RF. Differential expression of a novel gene, WDNM1, in nonmetastatic rat mammary adenocarcinoma cells. Cancer Res 1998;48:5203-5209.

46 Steeg PS. Search for metastasis suppressor genes. Invasion Metast 1989;9:351-359.

47 Aldaz CM, Hu Y, Daniel R, et al. Serial analysis of gene expression in normal p53 null mammary epithelium. Oncogene 2002;21:6366-6376.

48 Wellbrock C, Weisser C, Geissinger E, et al. Activation of p59fyn leads to melanocyte dedifferentiation by influencing MKP-1 regulated MAP kinase signalling. J Biol Chem 2001;277:6443-6454.

49 Kunapuli SP, Singh H, Singh P, et al. Ceruloplasmin gene expression in human cancer cells. Life Sci 1987;40:2225-2228.

50 Hough CD, Cho KR, Zonderman AB, et al. Coordinately up-regulated genes in ovarian cancer. Cancer Res 2001;61:3869-3876.

51 Ozyilkan O, Baltali E, Ozyilkan E, et al. Ceruloplasmin level in women with breast disease. Preliminary results. Acta Oncol 1992;31:843-846.

52 El Sewedy T, Fornaro M, Alberti S. Cloning of the murine TROP2 gene: conservation of a PIP2-binding sequence in the cytoplasmic domain of TROP-2. Int J Cancer 1998;75:324-330.

53 Ripani E, Sacchetti A, Corda D, et al. Human Trop-2 is a tumor-associated calcium signal transducer. Int J Cancer 1998;76:671-676.

54 Hajitou A, Sounni NE, Devy L, et al. Down-regulation of vascular endothelial growth factor by tissue inhibitor of metalloproteinase-2: effect on in vivo mammary 
tumor growth and angiogenesis. Cancer Res 2001;61: 3450-3457.

55 Taggart CC, Lowe GJ, Greene CM, et al. Cathepsin B, L, and $\mathrm{S}$ cleave and inactivate secretory leucoprotease inhibitor. J Biol Chem 2001;276:33345-33352.

56 Zhang Y, DeWitt DL, McNeely TB, et al. Secretory leukocyte protease inhibitor suppresses the production of monocyte prostaglandin $\mathrm{H}$ synthase-2, prostaglandin E2, and matrix metalloproteinases. J Clin Invest 1997;99:894-900.
57 Hartman DJ, Hoogenraad NJ, Condron R, et al. Identification of a mammalian $10-\mathrm{kDa}$ heat shock protein, a mitochondrial chaperonin 10 homologue essential for assisted folding of trimeric ornithine transcarbamoylase in vitro. Proc Natl Acad Sci USA 1992;89:3394-3398.

58 Quinn KA, Cavanagh AC, Hillyard NC, et al. Early pregnancy factor in liver regeneration after partial hepatectomy in rats: relationship with chaperonin 10. Hepatology 1994;20:1294-1302. 